TITLE:

\title{
Modeling spiking behavior of neurons with time-dependent Poisson processes
}

\section{$\operatorname{AUTHOR}(\mathrm{S})$ :}

Shinomoto, S; Tsubo, Y

\section{CITATION:}

Shinomoto, S ... [et al]. Modeling spiking behavior of neurons with timedependent Poisson processes. Physical Review E 2001, 64(4): 041910.

ISSUE DATE:

2001-10

URL:

http://hdl.handle.net/2433/50554

RIGHT:

Copyright 2001 American Physical Society 
PHYSICAL REVIEW E, VOLUME 64, 041910

\title{
Modeling spiking behavior of neurons with time-dependent Poisson processes
}

\author{
Shigeru Shinomoto* and Yasuhiro Tsubo ${ }^{\dagger}$ \\ Department of Physics, Graduate School of Science, Kyoto University, Sakyo-ku, Kyoto 606-8502, Japan
}

(Received 15 March 2001; revised manuscript received 9 May 2001; published 21 September 2001)

\begin{abstract}
Three kinds of interval statistics, as represented by the coefficient of variation, the skewness coefficient, and the correlation coefficient of consecutive intervals, are evaluated for three kinds of time-dependent Poisson processes: pulse regulated, sinusoidally regulated, and doubly stochastic. Among these three processes, the sinusoidally regulated and doubly stochastic Poisson processes, in the case when the spike rate varies slowly compared with the mean interval between spikes, are found to be consistent with the three statistical coefficients exhibited by data recorded from neurons in the prefrontal cortex of monkeys.
\end{abstract}

DOI: 10.1103/PhysRevE.64.041910

PACS number(s): 87.10.+e, 02.50.Ey

\section{INTRODUCTION}

Spike sequences recorded from cortical neurons in vivo are usually irregular. It is not established whether the standard neurospiking models can describe the experimentally observed spiking irregularity [1]. It has been asserted that the leaky integrate-and-fire model with temporally uncorrelated inputs is able to generate irregular spike sequences [2,3]. Motivated by this alleged finding, Shinomoto, Sakai, and Funahashi $[4,5]$ proposed examining the consistency of a neurospiking model with experimental spiking data by considering several statistical measures of the interspike intervals (ISIs): not only the coefficient of variation $C_{V}$, which measures the spiking irregularity, but also the skewness coefficient of the interval distribution, $S$, and the correlation coefficient of consecutive intervals, $R$.

A leaky integration process with temporally uncorrelated Gaussian input is termed an Ornstein-Uhlenbeck process (OUP). In this paper we refer to a threshold spike-reset process whose membrane dynamics are given by such a process as OUP. This OUP can generate an irregular spike sequence. The experimental spike sequences examined in Refs. [4,5] are in fact irregular (possessing large $C_{V}$ values), but the other two statistical coefficients $S$ and $R$ evaluated from them are distributed very widely beyond the range that any OUP can realize. The large $S$ values and the large $R$ values exhibited by some non-negligible percentage of neurons are the statistical characteristics of these experimental spike sequences that cannot be reproduced by any OUP, as explained in the following.

The first finding that leads us to reject the OUP as a model of biological spiking behavior concerns the incompatibility of their statistics in the $C_{V^{-}} S$ plane [4]. Among the experimental spike sequences examined in Refs. [4,5], the mean ISI is at least $30 \mathrm{msec}$ and typically greater than $100 \mathrm{msec}$. This is much larger than the membrane time constant, which is considered to range from 1 to $20 \mathrm{msec}$ [6]. Thus in any of the spiking sequences, model parameter values for which the mean ISI is less than the membrane time constant should be excluded from consideration. We always add this constraint

\footnotetext{
*Email address: shinomoto@ scphys.kyoto-u.ac.jp

${ }^{\dagger}$ Email address: tsubo@ton.scphys.kyoto-u.ac.jp
}

to the OUP when considering it as a neurospiking model. The feasible region of an OUP for infinite length sequences, given this constraint, is shown in Fig. 1(a) as the shaded area in the $C_{V}-S$ plane. The dashed line represents the envelope of the contours within which lie $99 \%$ of sequences containing 100 intervals generated by an OUP with the same constraint. The data taken from the monkey prefrontal cortex are overlaid in the same figure. Each dot represents statistics derived from 100 ISIs recorded from a neuron. The percentage of the experimental data lying outside this $1 \%$ envelope is $7.2 \%$. Those data that lie outside the $1 \%$ envelope do so because their $S$ values are too large, typically greater than 4 or 5 .

The second finding on which our rejection of the OUP is based is that regarding the correlation coefficient of consecutive intervals $R$. The $R$ values are expected to be distributed normally with mean zero and variance $1 / n$ for any spike sequences of $n$ intervals generated according to a renewal process, including an OUP and a Poisson process [7-9]. Here, the range within which lie $99 \%$ of sequences containing 100 intervals generated by a renewal process, corresponds to $R \in[-0.26,0.26]$, with $0.5 \%$ lying on either side of this range. This range is indicated by the vertical dashed lines in Fig. 1(b). The $R$ values obtained using the data recorded from the prefrontal cortical neurons are distributed with an overall shift to the positive side with respect to this range, and the percentage of the data lying outside the $1 \%$ deviation range is $11.3 \%$. This result implies that renewal processes in general, including the OUP, are incapable of describing the statistics of spiking behavior as reflected by the correlation coefficient $R$.

With these two results, the OUP alone was found to be unable to describe the experimental data. It was then shown, however, that the statistics of the experimental data could be reproduced by a leaky integrate-and-fire model that includes temporally correlated inputs, which are themselves generated by another Ornstein-Uhlenbeck process [5]. We refer to this model as a "colored OUP." In sweeping out all parameter values, however, the data generated by colored OUP occupies a much wider region in the space of the three statistical measures of interest than that of the experimental data. The colored OUP is therefore in some sense "overcapable" of describing neurospiking behaviors. One somewhat unnatural aspect of the behavior of the colored OUP is that it 
(a)

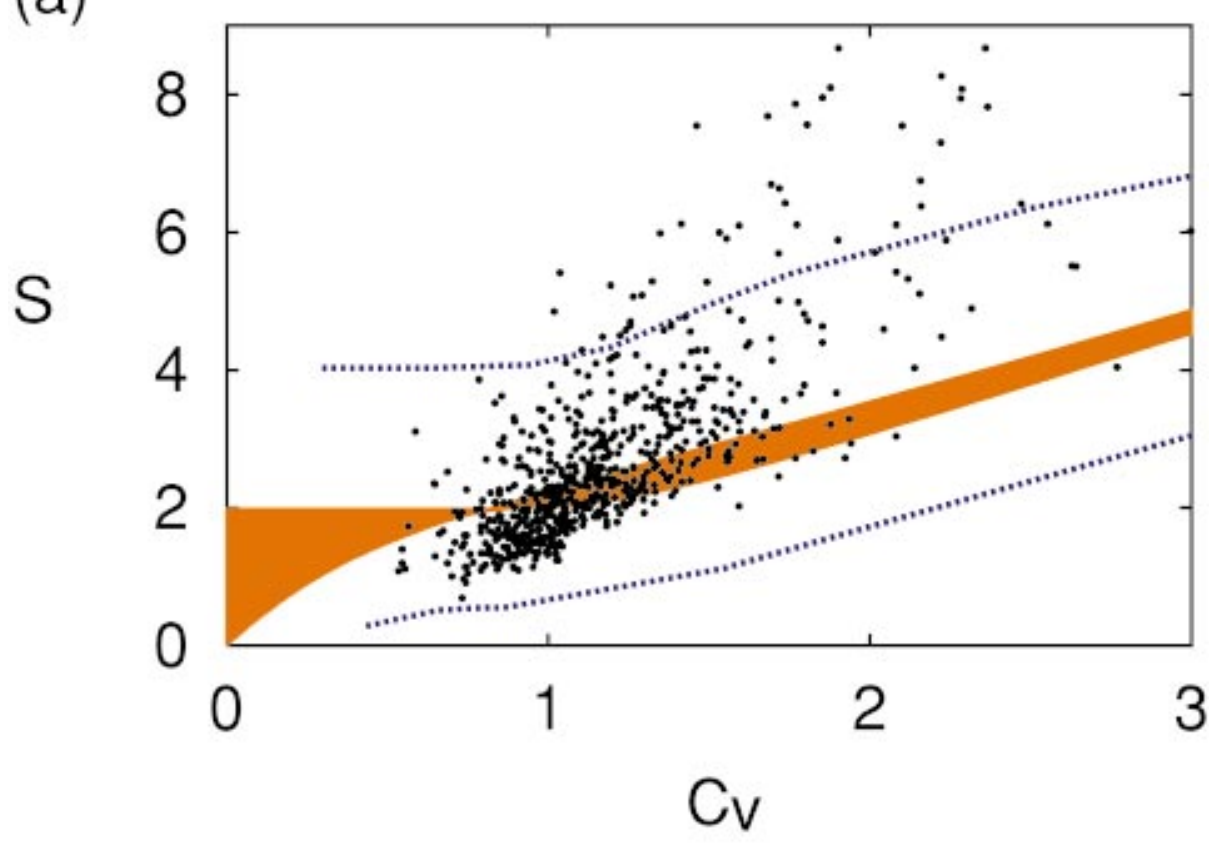

(b)

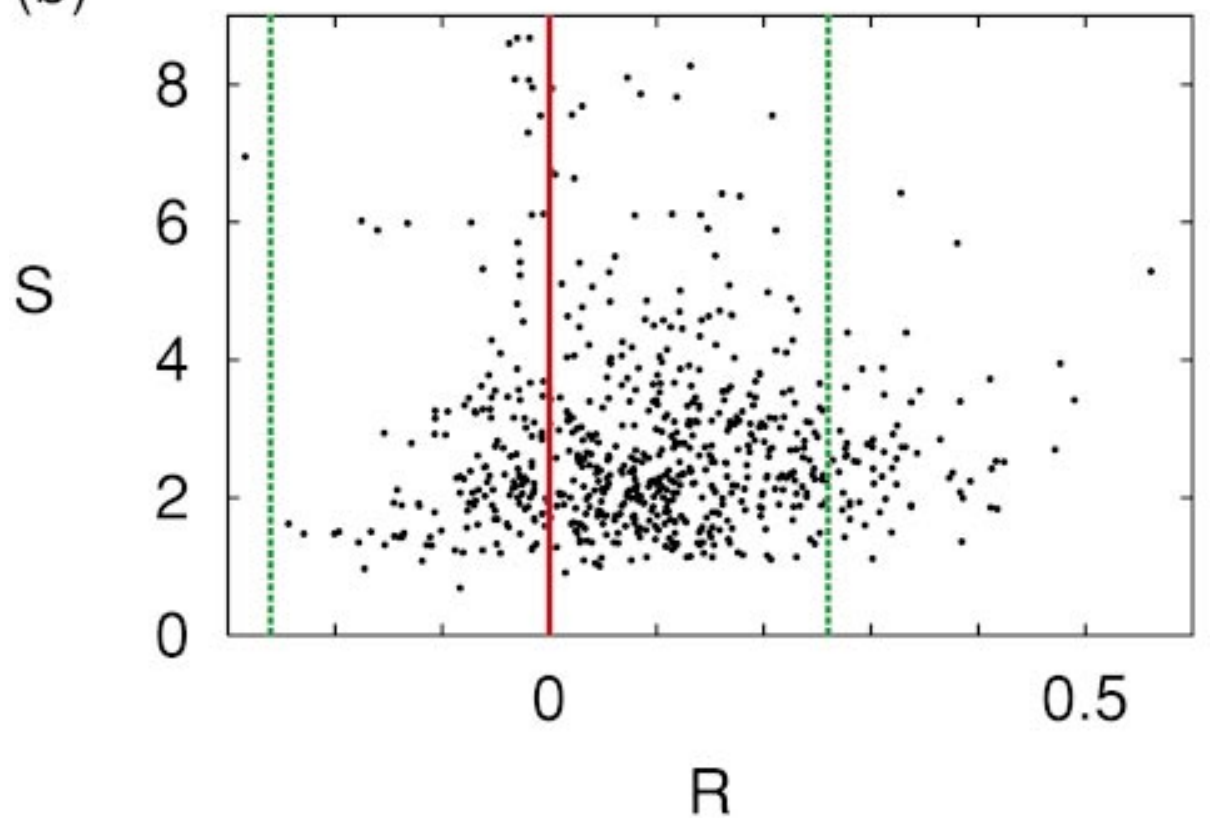

FIG. 1. (Color) Distributions of statistical coefficients for 666 spike sequences recorded from monkey prefrontal cortices (by courtesy of Shintaro Funahashi and Yutaka Sakai). Each dot represents a pair of statistical coefficients computed from a spike sequence consisting of 100 ISIs of a neuron. (a) The coefficient of variation $C_{V}$ and the skewness coefficient $S$. The shaded area represents the region that can be reproduced by the OUP with the constraint that the mean spike interval is greater than the membrane time constant. The dashed curves represent the envelope of the contours within which $99 \%$ of the data should exist given the finite number of intervals per sequence ( $n$ $=100$ ) if this spiking behavior were described by an OUP. The percentage of the data lying outside this $1 \%$ envelope is $7.2 \%$. This result clearly excludes the OUP as a possible model of such spiking behavior. (b) The correlation coefficient of consecutive intervals $R$ and the skewness coefficient $S$. Here, the range, within which lie $99 \%$ of sequences containing 100 ISIs generated by an OUP or any renewal process, corresponds to $R \in[-0.26,0.26]$, which is indicated by the vertical lines. The percentage of the experimental data lying outside these lines is $11.3 \%$. This result clearly excludes any renewal process. 
generates regular spike sequences over a fairly wide range of parameter values (The original OUP can also generate regular spike sequences). Although in actual biological systems, regular spiking can be observed in constant current injection experiments conducted in vitro $[10,11]$, in general it is not observed in the cortex of an active animal in vivo, and for this reason, there is no reason to require our model to be able to reproduce such behavior.

We wish to construct a simple model with a small number of parameters, which reproduces the spiking statistics of neurons in vivo without the necessity of fine tuning parameter values. As a possible type of such models, we study in the present paper time-dependent Poisson processes, in which point events (spikes) are generated randomly with a spike rate that is temporally modulated in some prescribed manner. We consider such models because we have proven that the coefficient of variation $C_{V}$ is greater than or equal to unity for a sequence of infinite length generated by any kind of time-dependent Poisson process. This fact appears to be consistent with experimental data of finite length taken in vivo. In the present paper we do not attempt a detailed examination of the consistency of such models with experimental data of finite length, but instead we obtain values for the three coefficients $C_{V}, S$, and $R$, describing the statistics of the spike sequences of infinite length, through analytic and numerical investigation for three types of time-dependent Poisson processes: pulse regulated, sinusoidally regulated, and doubly stochastic.

\section{THREE STATISTICAL COEFFICIENTS}

A sequence of point events (spikes) is registered in a form of a sequence of interevent intervals (ISIs) as $\left\{T_{1}\right.$, $\left.T_{2}, \cdots, T_{n}\right\}$, assuming the stationarity of the phenomena. The ISI sequence is characterized in the present paper by three statistical coefficients $C_{V}, S$, and $R$, which will be defined in this section. Theoretical statistical coefficients with respect to time-dependent Poisson processes will be computed for a sequence of infinite number of intervals. When performing the numerical simulation, the number of intervals $n$ was set very large $(n=1000000)$ so that the computed statistics can practically be regarded as theoretical values for $n=\infty$. With respect to the experimental ISI sequence, however, the number of available ISIs are limited, and we will fix the number of intervals as $n=100$ in computing the sample statistics.

The coefficient of variation $C_{V}$ is a measure of the variability of ISIs, defined as the ratio of the standard deviation to the mean,

$$
C_{V}={\overline{(T-\bar{T})^{2}}}^{1 / 2} / \bar{T} .
$$

Here $T$ is the interval and $\cdots$ represents an averaging operation such that $\bar{T} \equiv 1 / n \sum_{i=1}^{n} T_{i}$. The coefficient $C_{V}$ vanishes for a regular spike sequence. On the other hand, $C_{V}=1$ for an infinite length sequence generated by a fixed Poisson process. For a sequence of finite number of intervals $n$, the sample $C_{V}$ value is expected to be distributed about 1 with the deviation of the order of $1 / \sqrt{n}$, if the sequence is gener- ated by the Poisson process. We can see in Fig. 1(a) that most experimental spike data exhibit large values of $C_{V}$.

The skewness coefficient $S$ is a measure of the asymmetry of the interval distribution defined as

$$
S=\overline{(T-\bar{T})^{3}} / \overline{(T-\bar{T})^{2}}{ }^{3 / 2} .
$$

The skewness coefficient $S$ can be either positive or negative, but it is 2 for an infinite length sequence generated by the Poisson process. For a sequence of finite number of intervals $n$, the sample $S$ value is expected to be distributed about 2 with the deviation of the order of $1 / \sqrt{n}$, if the sequence is generated by the Poisson process. We can see in Fig. 1(a) that a large percentage of experimental data exhibit values of $S$ significantly larger than 2 .

The correlation coefficient $R$ is a measure of the mutual dependence of consecutive ISIs, defined as

$$
R=\left(\overline{T_{i} T_{i+1}}-\bar{T}^{2}\right) / \overline{(T-\bar{T})^{2}},
$$

where $T_{i}$ and $T_{i+1}$ denote a pair of consecutive intervals. The correlation coefficient $R$ vanishes for an infinite length sequence generated by any renewal process, including the OUP and the Poisson process. For a sequence of finite number of intervals $n$, the sample $R$ value is expected to be distributed normally about zero with variance $1 / n$ if the sequence is generated by a renewal process. As depicted in Fig. $1(b)$, a large percentage of the data taken from the monkey prefrontal cortex exhibit positive $R$ values significantly larger than what would result from the renewal process.

\section{THREE KINDS OF TIME-DEPENDENT POISSON PROCESSES}

In the Poisson process, point events occur randomly in time with a certain fixed rate $\lambda$. In this process, the interevent intervals $T$ appear as randomly and independently chosen values from an exponential probability distribution

$$
p(T)=\lambda e^{-\lambda T} .
$$

For an infinite length spike sequence generated by a fixed Poisson process, we can expect that the three statistical coefficients defined above will take the values $C_{V}=1, S=2$, and $R=0$. For a finite number of intervals so generated, the root mean square deviation from these values is of the order of $1 / \sqrt{n}$.

The values of the coefficients $C_{V}$ and $S$ evaluated from experimental spike sequences in fact have been found to be distributed around the values for the Poisson process, $C_{V}$ $=1$ and $S=2$. The extent to which this experimental data deviate from these values is, however, too large, given that each data point represents a sequence of 100 ISIs [4]. In addition, the values of $R$ found experimentally are as a whole shifted in the positive direction with respect to the range for the renewal process. For these reasons, a fixed Poisson process, in which the spike rate $\lambda$ is time independent, cannot reproduce the experimental spiking statistics. We therefore 
would like to consider extended Poisson processes in which the spike rate is given by some predefined functions of time $\lambda(t)$.

First, we consider a pulse regulated Poisson process, in which the spike rate is a periodic function consisting of Dirac $\delta$ functions separated by time intervals of length $s$,

$$
\lambda(t)=\sum_{k=-\infty}^{\infty} \nu \delta(t-s k) .
$$

Namely, multiple spikes (including no spike and one spike case) can be generated at each time $t=s k(k=\cdots, 0,1$, $2, \cdots)$. The number of spikes at each time is chosen according to the Poisson distribution with the mean $\nu$.

Second, we consider a sinusoidally regulated Poisson process, in which the spike rate undergoes a smooth periodic modulation as

$$
\lambda(t)=\lambda_{0}+\Delta \sin (t / s),
$$

where $\lambda_{0}(\geqslant|\Delta|)$ is the mean spike rate and $2 \pi s$ is the period of the modulation.

Third, we examine a doubly stochastic Poisson process in which the spike rate is randomly modulated $[7,8,12]$. We consider the case that the random modulation of the spike rate is given by the Ornstein-Uhlenbeck process,

$$
\frac{d \lambda}{d t}=-\frac{\lambda-\lambda_{0}}{s}+\sqrt{\Delta} \xi(t),
$$

where $\xi(t)$ is Gaussian white noise with ensemble-averaged quantities $\langle\xi(t)\rangle=0$ and $\left\langle\xi(t) \xi\left(t^{\prime}\right)\right\rangle=\delta\left(t-t^{\prime}\right)$. We introduced same notations $\Delta$ and $s$ as the abovementioned sinusoidally regulated Poisson process [Eq. (6)], as those parameters have mutually the same dimensionalities. This process yields the fluctuating spike rate

$$
\lambda(t)=\lambda_{0}+\Delta \eta(t),
$$

which is characterized by

$$
\langle\eta(t)\rangle=0,
$$

and

$$
\left\langle\eta(t) \eta\left(t^{\prime}\right)\right\rangle=e^{-\left|t-t^{\prime}\right| / s} .
$$

In this process we stipulate that spikes are not generated when $\lambda(t) \leqslant 0$.

\section{METHOD OF ANALYSIS}

In this section we introduce the method of obtaining theoretical moments of intervals, which constitute the three statistical coefficients for an infinite length sequence generated by the time-dependent Poisson process, in which point events (spikes) are generated randomly in time with the rate $\lambda(t)$. The conditional probability that given a spike at time $t$ the next spike will appear at time $t+T$ is [7-9]

$$
p(T \mid t)=\lambda(t+T) \exp \left[-\int_{0}^{T} d u \lambda(t+u)\right] .
$$

The $n$th moment of the ISI, when the preceding spike was generated at time $t$, is given by

$$
\left.\overline{T^{n}}\right|_{t}=\int_{0}^{\infty} d T T^{n} p(T \mid t) .
$$

The conditional moment of the ISIs averaged over all possible values of the time of the preceding spike is therefore given by

$$
\overline{T^{n}}=\lim _{U \rightarrow \infty} \frac{\left.\int_{0}^{U} d t \lambda(t) \overline{T^{n}}\right|_{t}}{\int_{0}^{U} d t \lambda(t)}=\lim _{U \rightarrow \infty} \frac{\int_{0}^{U} d t \lambda(t) \int_{0}^{\infty} d T T^{n} p(T \mid t)}{\int_{0}^{U} d t \lambda(t)} .
$$

This can be simplified by means of partial integration to yield the simple integral expressions

$$
\bar{T}=\frac{1}{\bar{\lambda}},
$$

$$
\begin{aligned}
& \overline{T^{2}}=\frac{2}{\bar{\lambda}} \int_{0}^{\infty} d T \overline{\exp \left[-\int_{0}^{T} d u \lambda(t+u)\right]}, \\
& \overline{T^{3}}=\frac{6}{\bar{\lambda}} \int_{0}^{\infty} d T T \overline{\exp \left[-\int_{0}^{T} d u \lambda(t+u)\right]},
\end{aligned}
$$

where $\bar{\cdots}$ on the right-hand side represents the long-time average defined as

$$
\bar{A}=\lim _{U \rightarrow \infty} \frac{1}{U} \int_{0}^{U} d t A(t),
$$

while $\cdots$ on the left-hand side represents the average over a long spike sequence defined in Sec. II.

The statistical coefficients $C_{V}$ and $S$ are given by the first few of the above moments as

$$
\begin{gathered}
C_{V}=\sqrt{\overline{T^{2}} / \bar{T}^{2}-1}, \\
S=\frac{\overline{T^{3}}-3 \overline{T^{2}} \bar{T}+2 \bar{T}^{3}}{\left(\bar{T}^{2}-\bar{T}^{2}\right)^{3 / 2}} .
\end{gathered}
$$

Evaluation of the correlation coefficient $R$ requires an average over two consecutive intervals, given as 


$$
\overline{T_{i} T_{i+1}}=\lim _{U \rightarrow \infty} \frac{\int_{0}^{U} d t \lambda(t) \int_{0}^{\infty} d T_{1} T_{1} p\left(T_{1} \mid t\right) \int_{0}^{\infty} d T_{2} T_{2} p\left(T_{2} \mid t+T_{1}\right)}{\int_{0}^{U} d t \lambda(t)} .
$$

Repeated use of partial integration (as explained in Appendix A) yields the simple form

$$
\begin{aligned}
\overline{T_{i} T_{i+1}}= & \frac{1}{\bar{\lambda}} \int_{0}^{\infty} d T_{1} \int_{0}^{\infty} d T_{2} \\
& \times \overline{\lambda\left(t+T_{1}\right) \exp \left[-\int_{0}^{T_{1}+T_{2}} d u \lambda(t+u)\right]} .
\end{aligned}
$$

With the quantity $\overline{T_{i} T_{i+1}}$ defined in this manner, the statistical coefficient $R$ is given by

$$
R=\frac{\overline{T_{i} T_{i+1}}-\bar{T}^{2}}{\overline{T^{2}}-\bar{T}^{2}} .
$$

\section{PROOF OF THE INEQUALITY $C_{V} \geqslant 1$}

It was pointed out by Tuckwell ( [9], pp. 222-225) that the relation $C_{V} \geqslant 1$ holds for a two-state semi-Markov process in which a spike generator alternates between two states with different spike rates. Here, we would like to prove that this inequality holds generally for any kind of timedependent Poisson process.

The inequality $C_{V} \geqslant 1$ is equivalent to

$$
\bar{T}^{2} \geqslant 2 \bar{T}^{2},
$$

or in other words,

$$
\frac{\bar{T}^{2}}{2 \bar{T}} \geqslant \bar{T} .
$$

The left-hand side of this relation is

$$
\int_{0}^{\infty} d T \overline{\exp \left[-\int_{0}^{T} d u \lambda(t+u)\right]}
$$

and its integrand satisfies Jensen's inequality (see, for instance, Ref. [13]),

$$
\overline{\exp \left[-\int_{0}^{T} d u \lambda(t+u)\right]} \geqslant \exp \overline{\left[-\int_{0}^{T} d u \lambda(t+u)\right]}=e^{-T \bar{\lambda}}
$$

Using this, we can prove Eq. (15),

$$
\begin{aligned}
\frac{\overline{T^{2}}}{2 \bar{T}} & =\int_{0}^{\infty} d T \overline{\exp \left[-\int_{0}^{T} d u \lambda(t+u)\right]} \\
& \geqslant \int_{0}^{\infty} d T \exp (-T \bar{\lambda})=\frac{1}{\bar{\lambda}}=\bar{T} .
\end{aligned}
$$

Namely, the coefficient of variation $C_{V}$ is larger than or equal to unity for a spike sequence of infinite length generated by any kind of time-dependent Poisson process. The equality holds if the spike rate is constant in time, that is, in the case of a fixed Poisson process.

\section{EVALUATION OF INTERVAL STATISTICS}

In this section, we evaluate the three statistical coefficients $C_{V}, S$, and $R$ analytically and also report the results of their numerical evaluation for the three kinds of timedependent Poisson processes defined above: pulse regulated, sinusoidally regulated, and doubly stochastic. Among those three processes, the first two are not stationary. If the statistical coefficients are evaluated in a finite interval of time for those nonstationary processes, they are generally not invariant with respect to the shift of time. But if the number of intervals $n$ used for the evaluation is sufficiently large to overcome the nonstationary time scale, the statistical coefficients evaluated in this section for infinite length sequences are expected to be close to those for finite length sequences. The obtained results are compared with the experimental data displayed in Fig. 1 to elucidate the potentiality of the respective models for describing neuronal spiking behavior in vivo.

\section{A. Pulse regulated Poisson process}

The pulse regulated Poisson process, whose spike rate is expressed by Eq. (5), is analytically tractable. Though this modulation is periodic, the spiking behavior it generates differs significantly from that in the sinusoidally regulated case. As the spiking probability is given by the Dirac $\delta$ function, the model tends to generate multiple spikes at a single time. This is reminiscent of the spiking behavior of intrinsic bursting cells and chattering cells, both of which generate bursts of several successive spikes that appear at intervals of a few milliseconds, while the interburst interval is typically of the order of several tens of milliseconds or several hundreds of milliseconds [14].

The probability of the number of spikes $n$ generated at each time at which the argument of the delta function of Eq. (5) vanishes $(t=\cdots, 0, s, 2 s, 3 s, \cdots)$ is distributed according to the Poisson distribution 


$$
p_{n}=\frac{\nu^{n}}{n !} e^{-\nu} \text {. }
$$

The statistical quantities necessary to derive $C_{V}, S$, and $R$ are readily derived from this probability distribution as

$$
\begin{gathered}
\bar{T}=\frac{s}{\nu}, \\
\overline{T^{2}}=\frac{s^{2}}{\nu}\left(\frac{1+e^{-\nu}}{1-e^{-\nu}}\right), \\
\overline{T^{3}}=\frac{s^{3}}{\nu}\left[\frac{1+4 e^{-\nu}+e^{-2 \nu}}{\left(1-e^{-\nu}\right)^{2}}\right], \\
\overline{T_{i} T_{i+1}}=s^{2}\left[\frac{e^{-\nu}}{\left(1-e^{-\nu}\right)^{2}}\right] .
\end{gathered}
$$

We thus see that the ISI depends linearly on $s$. For this reason, nondimensional spiking statistics such as $C_{V}, S$, and $R$ do not depend on $s$ but only on $\nu$.

Figure 2 displays statistical coefficients for the pulse regulated Poisson process. In the limit of $\nu \rightarrow 0$, the values of the statistical coefficients converge to their Poisson values, $C_{V}$ $=1, S=2$, and $R=0$. Negative values of $R$ and small values of $S$ are two main characteristics of this process that emerge as $\nu$ is increased from 0 . In this pulse regulated case, $S$ asymptotically approaches the line $S=C_{V}$ for large $\nu$. This behavior, however, is not consistent with the experimental data displayed in Fig. 1. In that figure, we see that a large number of the data points have values of $S$ that are distributed well above the $S=C_{V}$ line. In addition, the values of $R$ generated by this model are inconsistent with the experimental values, as the former are shifted in the negative direction with respect to 0 , while the latter are shifted in the positive direction. From these results, we see that the spike sequences recorded from monkey prefrontal cortical neurons cannot be described by the pulse regulated Poisson process.

\section{B. Sinusoidally regulated Poisson process}

The activity of the neurons of alert animals may be temporally modulated by brain waves. Types of brain waves are classified according to their frequency range as $\alpha$ waves $(8-13 \mathrm{~Hz}), \beta$ waves $(13-30 \mathrm{~Hz}), \theta$ waves $(4-7 \mathrm{~Hz})$, and $\delta$ waves $(0.5-4 \mathrm{~Hz})$ [15]. As a simple model to examine the effects of a smooth periodic modulation of this kind, we consider here the sinusoidally regulated Poisson process discussed briefly above, whose spike rate is given by Eq. (6). We note here that sinusoidally regulated noisy leaky integrate-and-fire models have been studied by Lansky [16] and Bulsara et al. [17] in different contexts, related to stochastic resonance phenomena.

In the present case of a periodically modulated spike rate, the "long-time" average

$$
\bar{A}=\lim _{U \rightarrow \infty} \frac{1}{U} \int_{0}^{U} d t A(t)
$$

can be replaced by the time average over one period of the modulation,

$$
\bar{A}=\frac{1}{2 \pi s} \int_{0}^{2 \pi s} d t A(t) .
$$

This replacement enables us to perform numerical integration of Eqs. (11), (12), and (14) to obtain $\overline{T^{2}}, \overline{T^{3}}$, and $\overline{T_{i} T_{i+1}}$, which yield the quantities $C_{V}, S$, and $R$.

The value of these quantities obtained by analytic integration are valid for $\lambda_{0}>\Delta$. The results obtained with this direct integration method are depicted in Fig. 3. We have also performed numerical simulations of the original stochastic model with various parameters, but we do not include the simulation results in the figures, as they essentially coincide with those obtained from the numerical integration method.

If the modulation of the spike rate is much more rapid than the mean spike rate $\left(s \ll 1 / \lambda_{0}\right)$, the coefficients $C_{V}, S$, and $R$ take on their values for a Poisson process. In this regular modulation, it would be possible to catch the rapid regular modulation by means of the other kind of statistics such as the autocorrelation function, but we are presently not interested in those statistics. As seen in the figure, the value of $C_{V}$ increases as $s$ is increased from 0 , while the values of $S$ and $R$ first decrease from the Poisson values 2 and 0 , respectively, and then reverse, eventually exceeding these values as $s$ approaches the order of the mean interval $1 / \lambda_{0}$. The large values of $C_{V}, S$, and $R$ produced for large modulation $\Delta / \lambda_{0}$ of long-time scale $s \lambda_{0}$ reproduce the experimental data displayed in Fig. 1, but the values obtained for values of the small $s \lambda_{0}$ do not correspond well with the experimental data.

\section{The doubly stochastic Poisson process}

Even if a neuron generates spikes randomly in time, it would also be subject to slowly fluctuating current and the resulting spike rate may fluctuate accordingly in time. As a simple model to express the random temporal fluctuation, we would like to consider the doubly stochastic Poisson process, whose spike rate is given by Eq. (7),

$$
\lambda(t)=\lambda_{0}+\Delta \eta(t),
$$

where $\eta$ is the correlated Gaussian noise with the ensemble characteristics $\langle\eta(t)\rangle=0$ and $\left\langle\eta(t) \eta\left(t^{\prime}\right)\right\rangle=\exp \left(-\left|t-t^{\prime}\right| / s\right)$.

Here we assume that the ergodicity and thus the long-time average over one sample,

$$
\bar{A}=\lim _{U \rightarrow \infty} \frac{1}{U} \int_{0}^{U} d t A(t),
$$

can be replaced by the ensemble average $\langle A\rangle$ over the Gaussian distribution of the ensemble of $\eta(t)$. In this Gaussian model, $\lambda(t)$ is distributed normally about $\lambda_{0}$ with deviation of order $\Delta$. Accordingly, $\lambda(t)$ can take negative values 
(a)

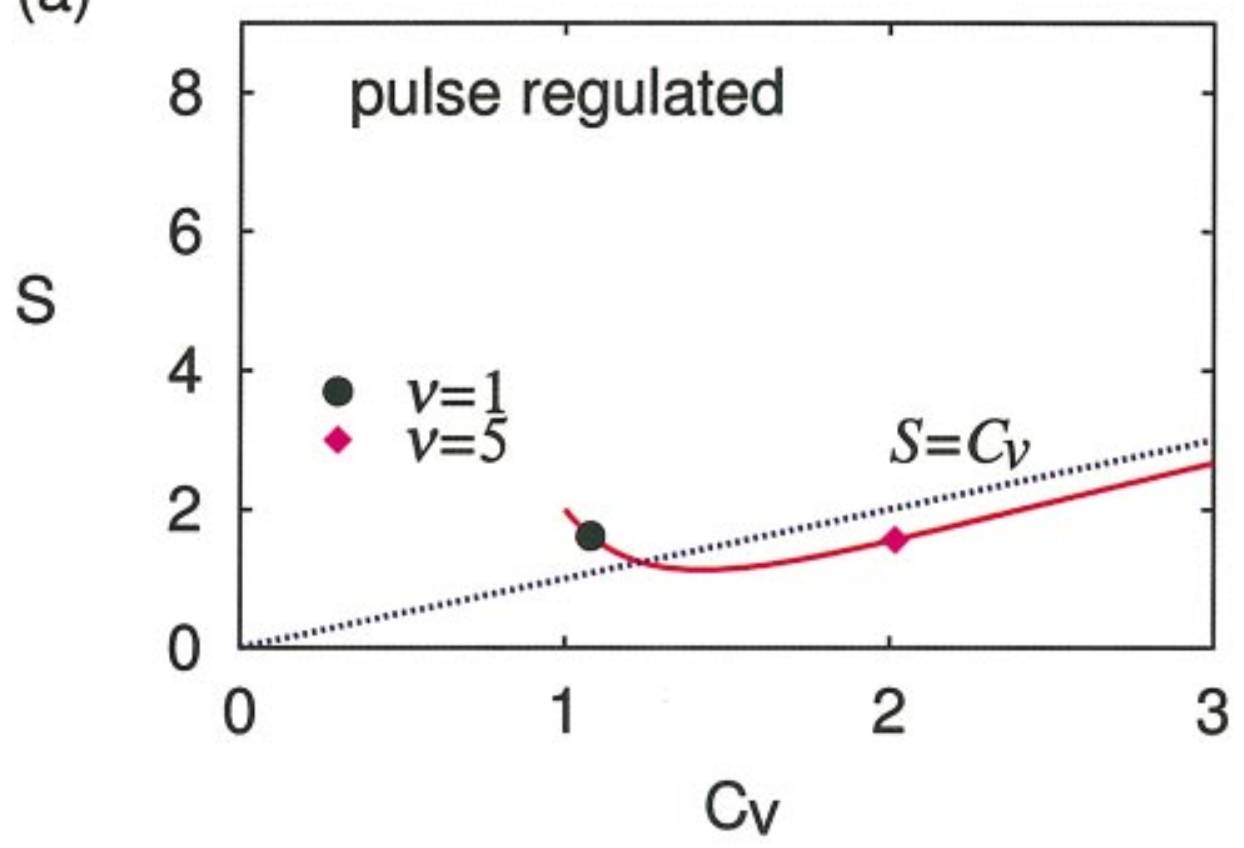

(b)

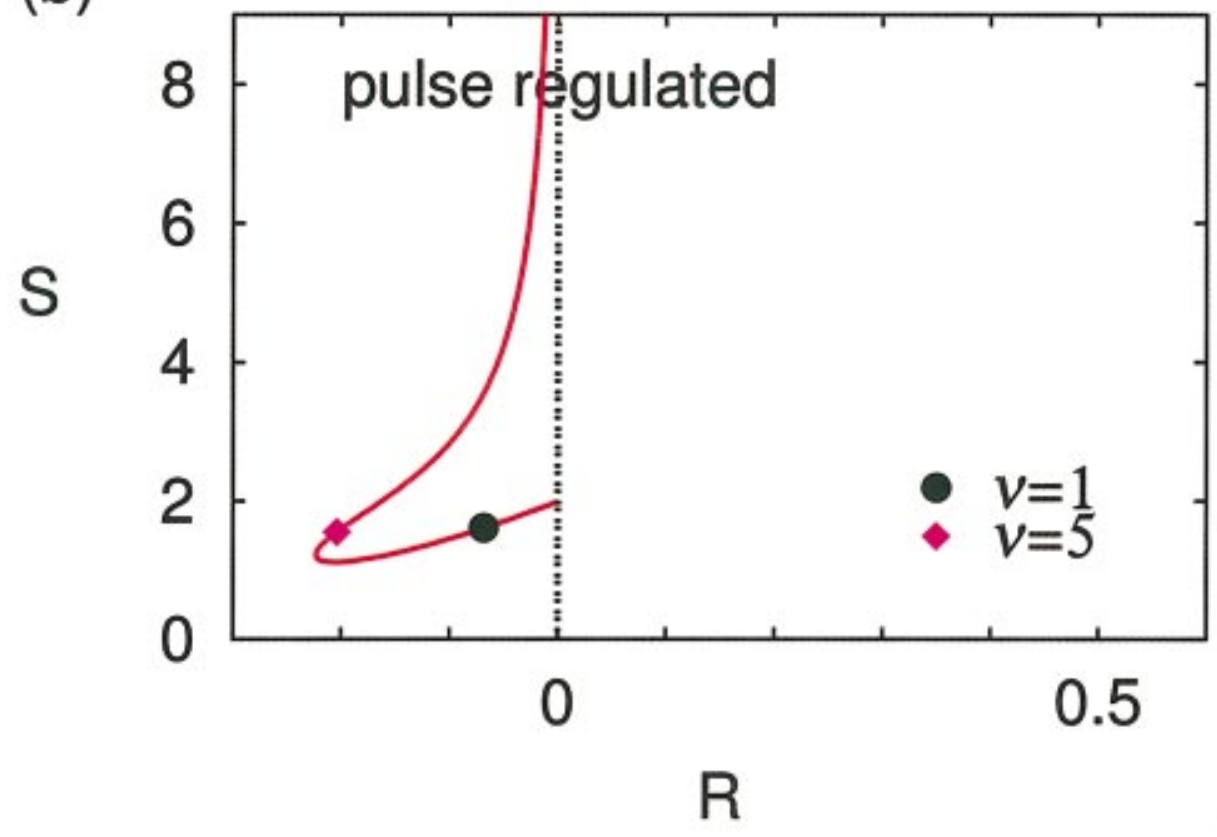

FIG. 2. (Color) Pulse regulated Poisson process. The statistical coefficients $C_{V}, S$, and $R$ depend only on $\nu$. In the limit $\nu \rightarrow 0$, the statistical coefficients converge to the Poisson values $C_{V}=1$, $S=2$, and $R=0$. (a) The $C_{V^{-}} S$ plane. We see that $C_{V}$ increases monotonically as a function of $\nu$, while $S$ first decreases and then asymptotically approaches the dashed line representing $S=C_{V}$. (b) The $R-S$ plane. Here we see that $R$ remains negative for all $\nu$. and this results in errors in the analysis. The analytical results can be considered reliable for $2 s \Delta^{2} \ll \lambda_{0}$.

The mean interval value is given simply by

$$
\bar{T}=\frac{1}{\lambda_{0}}
$$

and the higher moments $(n \geqslant 2)$ are given by

$$
\bar{T}^{n}=\frac{n(n-1)}{\lambda_{0}} \int_{0}^{\infty} d T T^{n-2} e^{-\lambda_{0} T}\left\langle\exp \left[-\Delta \int_{0}^{T} d u \eta(u)\right]\right\rangle .
$$

The ensemble average with respect to the Gaussian distribution of $\eta(t)$ is analytically obtained as (see Appendix B)

$$
\left\langle\exp \left[-\Delta \int_{0}^{T} d u \eta(u)\right]\right\rangle=e^{f(T)}
$$


(a)

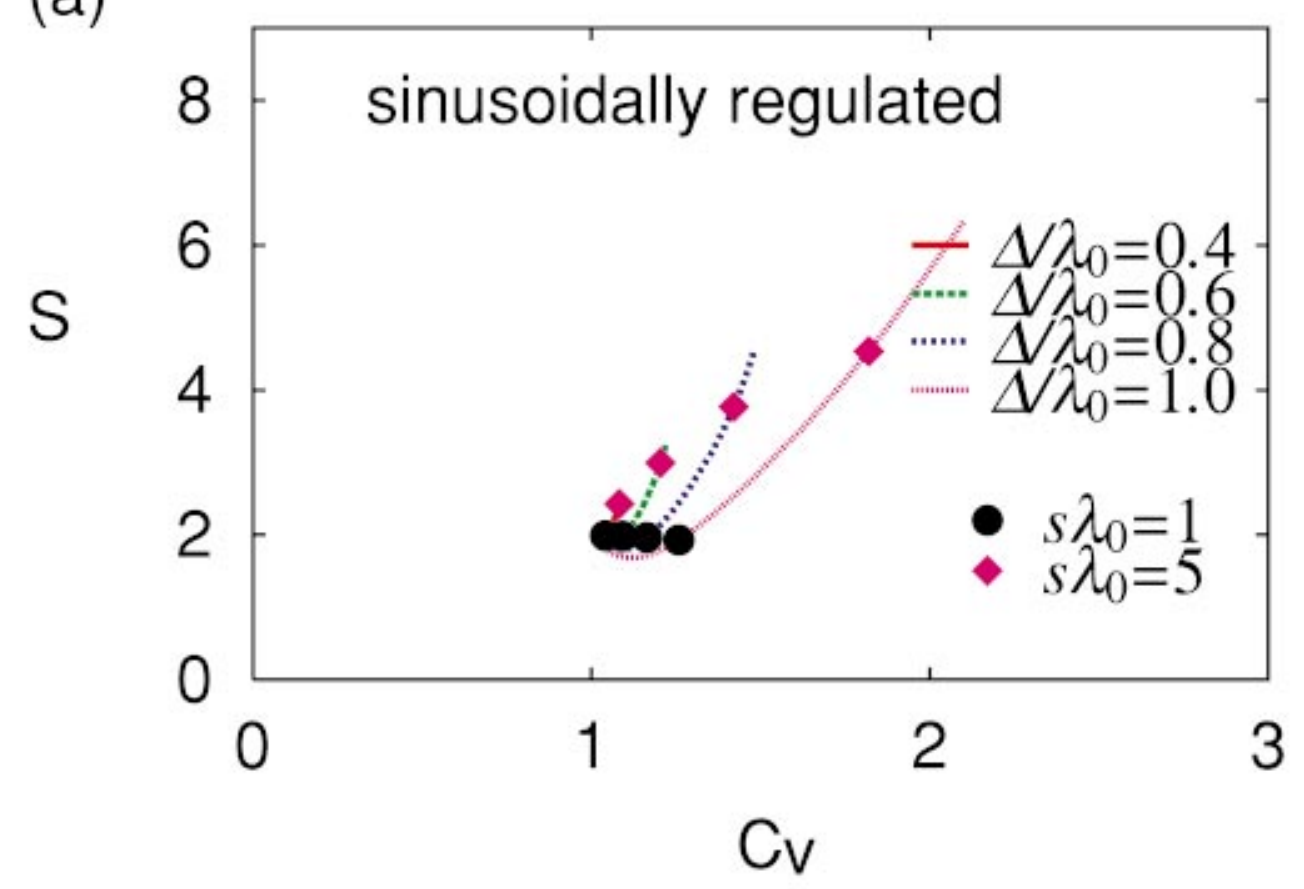

(b)

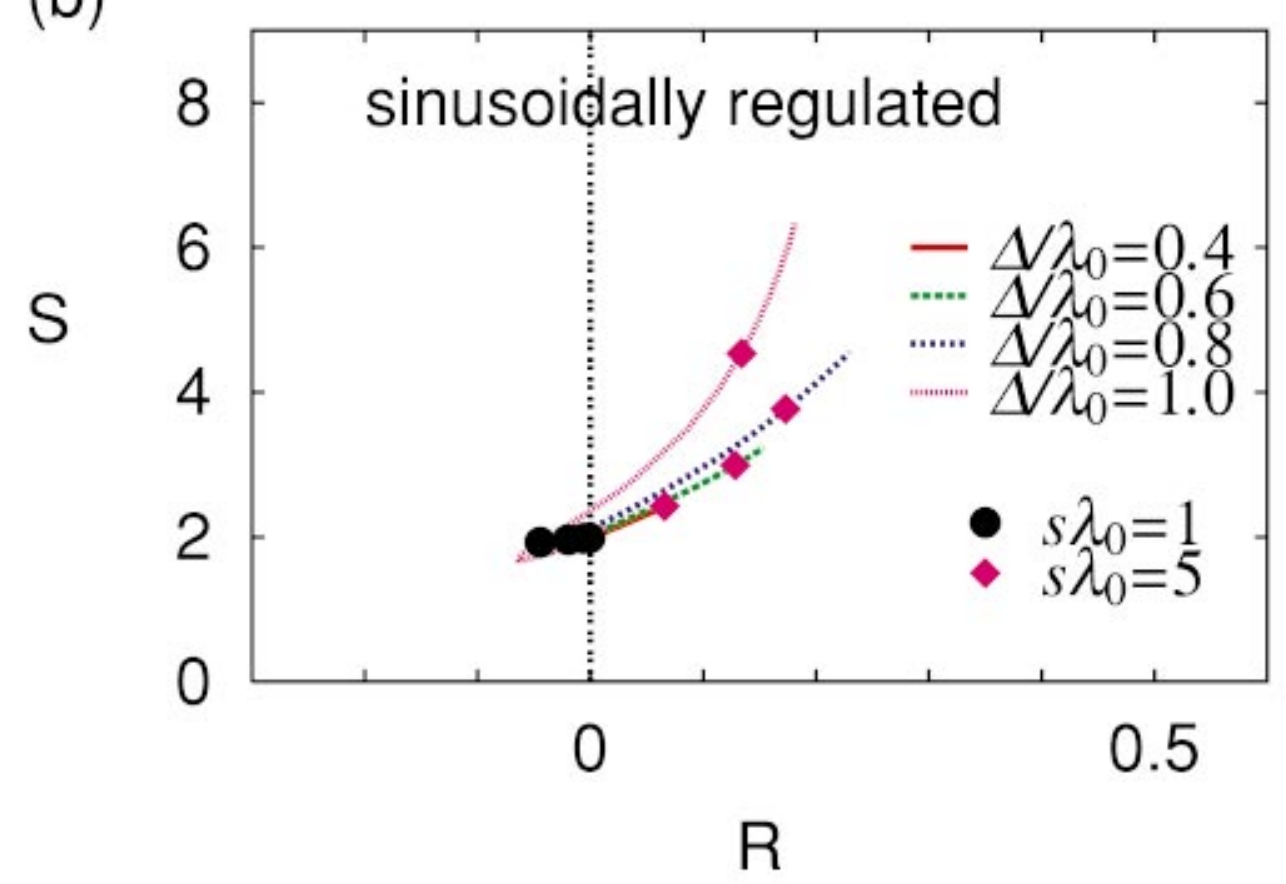

FIG. 3. (Color) Sinusoidally regulated Poisson process: In this case the statistical coefficients $C_{V}, S$, and $R$ depend on two parameters, $\Delta / \lambda_{0}$ and $s \lambda_{0}$. In either limit $\Delta \rightarrow 0$ or $s \rightarrow 0$, the statistical coefficients converge to the Poisson values, $C_{V}=1, S=2$, and $R=0$. The curves represent the iso- $\Delta$ curves $\left(\Delta=0.4,0.6,0.8\right.$, and 1.0). (a) The $C_{V^{-}} S$ plane. We see that $S$ first decreases from the Poisson value 2 as $s$ is increased from 0 , and then it reverses its course as $s$ approaches the order of the mean interval $\lambda_{0}^{-1}$, while $C_{V}$ increases monotonically with $s$. (b) The $R-S$ plane. Here it is seen that $R$ first decreases from the Poisson value 0 as $s$ is increased from 0 , and then it reverses its course as $s$ approaches the order of $\lambda_{0}^{-1}$. 


$$
f(T)=\Delta^{2} s^{2}\left(T / s-1+e^{-T / s}\right),
$$

and the final analytical integral form of the moments is given by

$$
\overline{T^{n}}=\frac{n(n-1)}{\lambda_{0}} \int_{0}^{\infty} d T T^{n-2} e^{-\lambda_{0} T+f(T)} .
$$

This integral formula is used for the evaluation of statistical coefficients $C_{V}$ and $S$. It should be noted that the moments given in Eq. (20) can, in principle, be derived from the probability distribution of intervals given by Cox and Lewis [7], (pp. 179-183). But the straightforward method described in Appendix B enables us to derive the following further results.

First, the average over the consecutive intervals is written as the ensemble average,

$$
\begin{aligned}
\overline{T_{i} T_{i+1}}= & \frac{1}{\lambda_{0}} \int_{0}^{\infty} d T_{1} \int_{0}^{\infty} d T_{2} \\
& \times\left\langle\lambda\left(T_{1}\right) \exp \left[-\int_{0}^{T_{1}+T_{2}} d u \lambda(u)\right]\right\rangle .
\end{aligned}
$$

The integrand on the right-hand side is carried out by means of the functional derivative, as explained in Appendix B, and we arrive at the final single integral form of the correlation,

$$
\overline{T_{i} T_{i+1}}=\frac{1}{\lambda_{0}} \int_{0}^{\infty} d T\left[\lambda_{0} T-2 f(T)\right] e^{-\lambda_{0} T+f(T)} .
$$

This formula is used for the evaluation of the correlation coefficient $R$.

The statistical coefficients $C_{V}, S$, and $R$ evaluated by the analytical integration of the integrals in Eqs. (20) and (22) are plotted in Fig. 4. If the modulation of the spiking probability fluctuates very rapidly in comparison with the mean spike rate $\left(s \ll 1 / \lambda_{0}\right)$, as seen in the figure, this modulation does not alter the statistics from that obtained for the Poisson process, $C_{V}=1, S=2$, and $R=0$.

The values of the statistical coefficients obtained by performing the numerical simulation of the original dynamical equations are displayed in Fig. 5. We find that the analytical results are in agreement with the simulation results only for small $s \Delta^{2} / \lambda_{0}$. It is thus concluded that numerical simulations are necessary to determine the general behavior of the statistical coefficients in this case. An important point regarding the present results is that the values of $S$ significantly larger than 2 and the positive $R$ values, which are exhibited by some significant percentage of the experimental data recorded from the monkey prefrontal cortex (Fig. 1), can be reproduced by the present doubly stochastic Poisson process in the case when $\Delta$ is larger than $0.5 \lambda_{0}$ and $s$ is larger than $1 / \lambda_{0}$, that is, with a fairly large modulation and slow time scale.

\section{CONCLUSION}

In the present paper, we have obtained values for three quantities describing interval statistics for three kinds of time-dependent Poisson processes: pulse regulated, sinusoidally regulated, and doubly stochastic. The values of these quantities obtained for each process were compared with distributions of the data recorded from the monkey prefrontal cortex. We find that among these three processes, the doubly stochastic Poisson process corresponds best to the experimental data. It would be interesting to perform more detailed statistical tests to examine the consistency of this model with the experimental data. It is also desirable to examine how ISI statistics depend on the area of the brain from which they are taken, as well as on the nature of the individual neuron used. We are proceeding with this study in these directions.

\section{ACKNOWLEDGMENTS}

Thanks are due to Shintaro Funahashi and Yutaka Sakai, respectively, for providing the original experimental data and the processed data, which were used in preparing Fig. 1. This study is supported in part by Grants-in-Aid for Scientific Research (No. 13210073 and No. 12680382) to S.S. by the Ministry of Education, Culture, Sports, Science and Technology, Japan.

\section{APPENDIX A}

In this appendix, we derive Eq. (14) from Eq. (13). Using the relation

$$
\begin{aligned}
& \frac{\partial}{\partial T_{2}} \exp \left[-\int_{0}^{T_{1}+T_{2}} d u \lambda(t+u)\right] \\
& \quad=-\lambda\left(t+T_{1}+T_{2}\right) \exp \left[-\int_{0}^{T_{1}+T_{2}} d u \lambda(t+u)\right],
\end{aligned}
$$

the numerator of Eq. (13) can be simplified as

$$
\begin{gathered}
\int_{0}^{U} d t \int_{0}^{\infty} d T_{1} \int_{0}^{\infty} d T_{2} \lambda(t) T_{1} \lambda\left(t+T_{1}\right) T_{2} \lambda\left(t+T_{1}+T_{2}\right) \exp \left[-\int_{0}^{T_{1}+T_{2}} d u \lambda(t+u)\right] \\
=\int_{0}^{U} d t \int_{0}^{\infty} d T_{1} \int_{0}^{\infty} d T_{2} \lambda(t) T_{1} \lambda\left(t+T_{1}\right) \exp \left[-\int_{0}^{T_{1}+T_{2}} d u \lambda(t+u)\right]
\end{gathered}
$$

We then carry out another partial integration, using the relation 
(a)

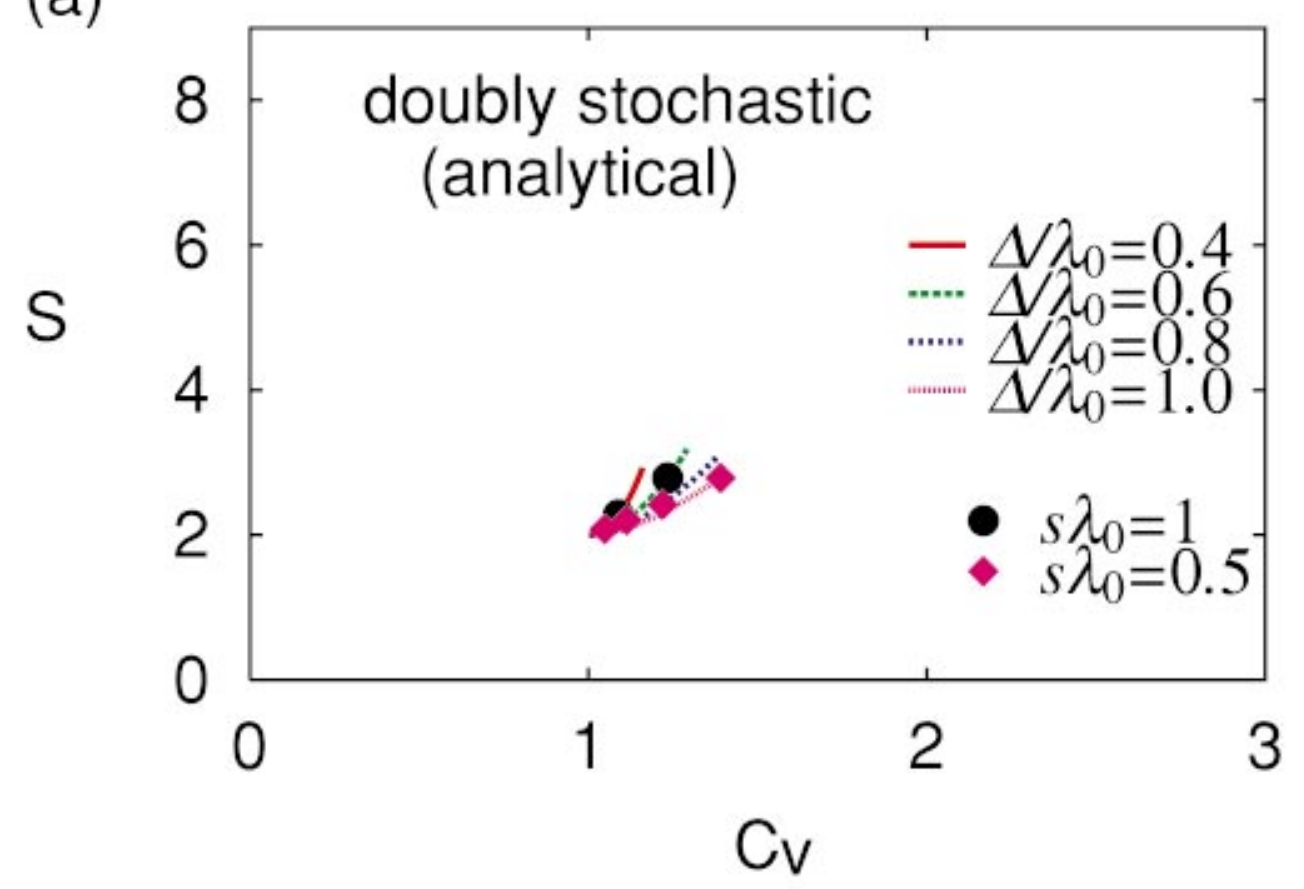

(b)

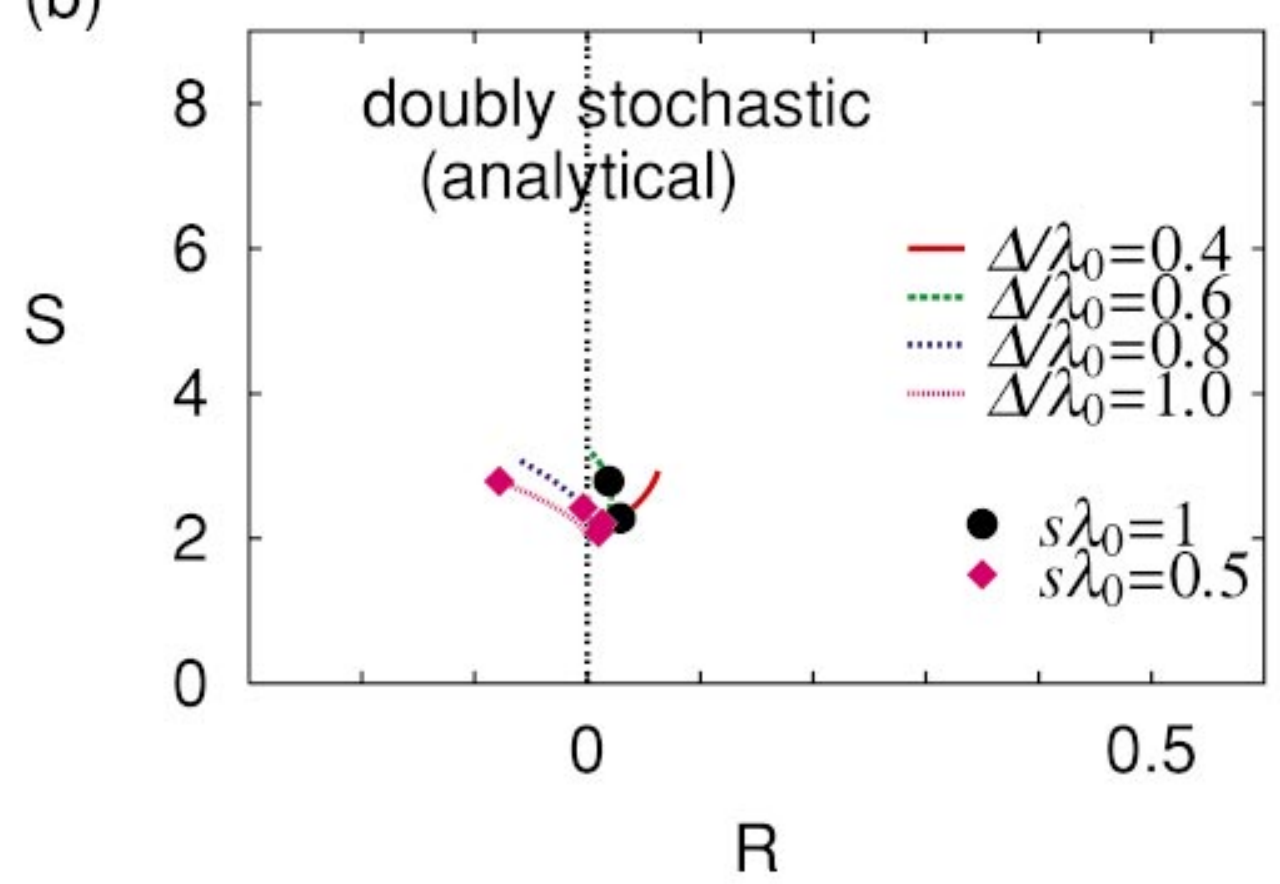

FIG. 4. (Color) Doubly stochastic Poisson process (analytical integration). Here too, the statistical coefficients $C_{V}, S$, and $R$ depend on the two parameters, $\Delta / \lambda_{0}$ and $s \lambda_{0}$. Once again, in either limit $\Delta \rightarrow 0$ or $s \rightarrow 0$, the statistical coefficients converge to the Poisson values $C_{V}=1, S=2$, and $R=0$. From the results of numerical simulations, however, we find that these analytical results are only reliable for $2 s \Delta^{2} \ll \lambda_{0}$. The curves represent the iso- $\Delta$ curves $\left(\Delta=0.4,0.6,0.8\right.$, and 1.0). (a) The $C_{V^{-}} S$ plane. Here we see that both $C_{V}$ and $S$ increase as $s$ is increased from 0 . (b) The $R-S$ plane. Here, $R$ also increases from the Poisson value 0 as $s$ is increased from 0 . 
(a)

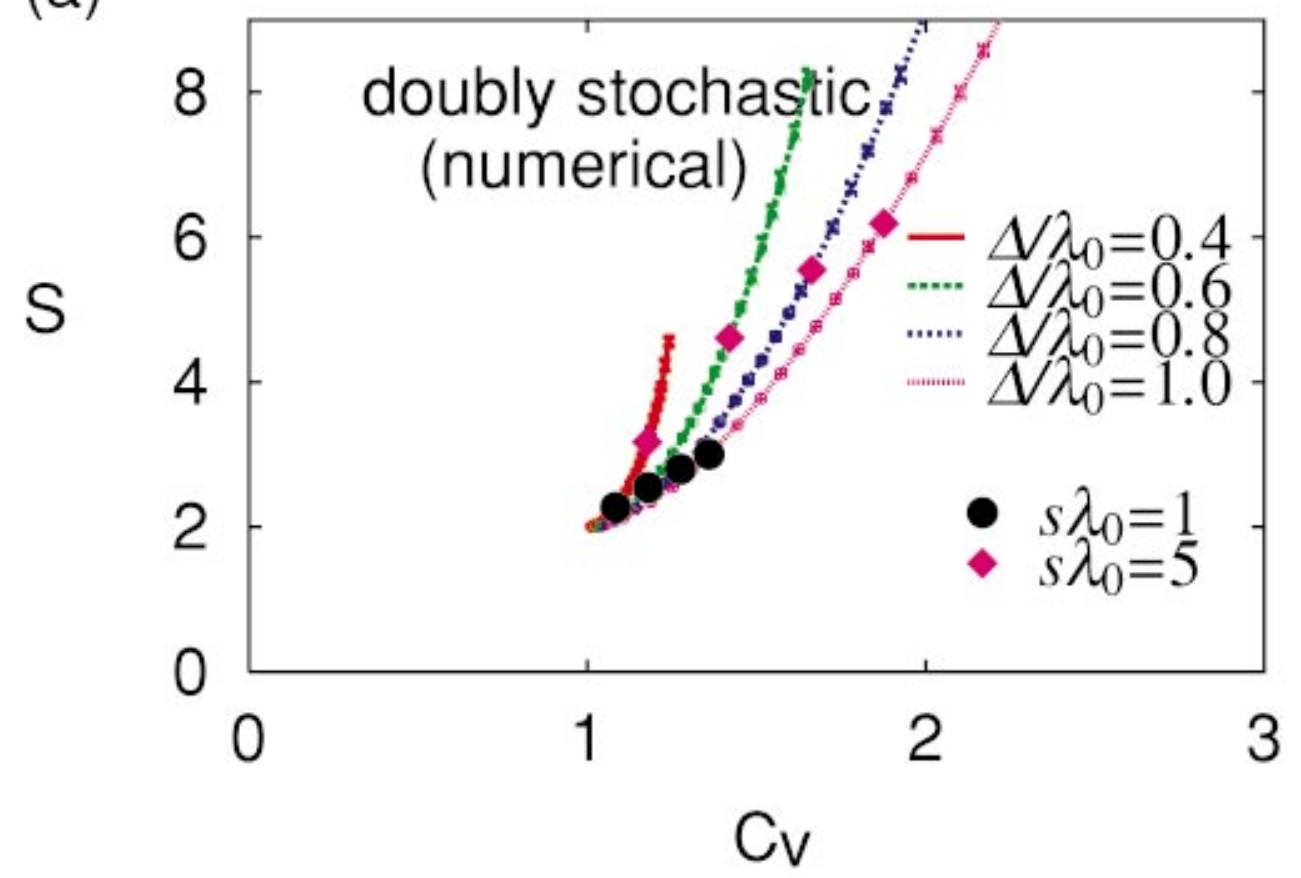

(b)

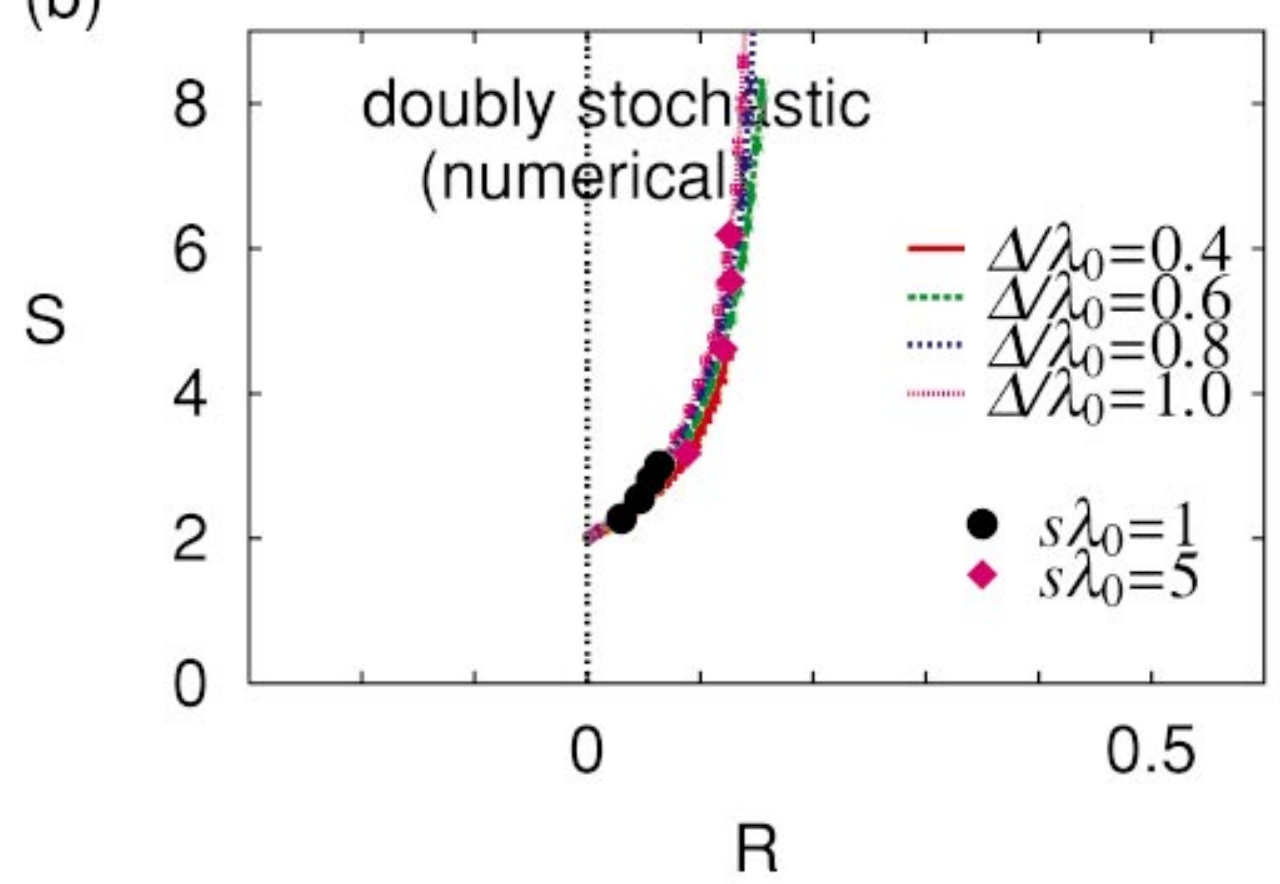

FIG. 5. (Color) Doubly stochastic Poisson process (numerical simulation), Each statistical coefficient is evaluated from a sequence of 1000000 numerically generated intervals. The curves represent the iso- $\Delta$ curves $(\Delta=0.4,0.6,0.8$, and 1.0$)$. (a) The $C_{V^{-}} S$ plane. (b) The $R-S$ plane. The departure of the analytical results from this numerical simulation results becomes significant as $s$ becomes comparable to $\Delta^{2} \lambda_{0}$. In this case, the present numerical results are more reliable. 


$$
\frac{\partial}{\partial t} \exp \left[-\int_{0}^{T} d u \lambda(t+u)\right]=[\lambda(t)-\lambda(t+T)] \exp \left[-\int_{0}^{T} d u \lambda(t+u)\right],
$$

where $T=T_{1}+T_{2}$. Ignoring the $O(1)$ end effects and retaining only the bulk integration, the numerator can be rewritten as

$$
\int_{0}^{U} d t \int_{0}^{\infty} d T_{1} \int_{0}^{\infty} d T_{2} T_{1} \lambda\left(t+T_{1}\right) \lambda\left(t+T_{1}+T_{2}\right) \exp \left[-\int_{0}^{T_{1}+T_{2}} d u \lambda(t+u)\right]+O(1)
$$

Repeating this kind of partial integration, neglecting end effects of $O(1)$, we arrive at

$$
\begin{aligned}
& \int_{0}^{U} d t \int_{0}^{\infty} d T_{1} \int_{0}^{\infty} d T_{2} \lambda\left(t+T_{1}\right) \exp \left[-\int_{0}^{T_{1}+T_{2}} d u \lambda(t+u)\right] \\
& \quad+O(1) .
\end{aligned}
$$

This gives the final formula, Eq. (14),

$$
\begin{aligned}
\overline{T_{i} T_{i+1}}= & \frac{1}{\bar{\lambda}} \int_{0}^{\infty} d T_{1} \int_{0}^{\infty} d T_{2} \\
& \times \lambda\left(t+T_{1}\right) \exp \left[-\int_{0}^{T_{1}+T_{2}} d u \lambda(t+u)\right]
\end{aligned}
$$

\section{APPENDIX B}

In this appendix, we perform the Gaussian integral to obtain Eqs. (19) and (22). First, the average

$$
\left\langle\exp \left[-\Delta \int_{0}^{T} d u \eta(u)\right]\right\rangle
$$

taken over the Gaussian distribution of $\eta(t)$, with $\langle\eta(t)\rangle$ $=0$ and $\left\langle\eta(t) \eta\left(t^{\prime}\right)\right\rangle=\exp \left(-\left|t-t^{\prime}\right| / s\right)$ is readily obtained as

$$
\begin{aligned}
& \left\langle\exp \left[-\Delta \int_{0}^{T} d u \eta(u)\right]\right\rangle \\
& =\exp \left[\frac{1}{2} \Delta^{2} \int_{0}^{T} d u \int_{0}^{T} d v\langle\eta(u) \eta(v)\rangle\right] \\
& =\exp \left[\Delta^{2} \int_{0}^{T} d u(T-u) e^{-u / s}\right]
\end{aligned}
$$

and this finally leads to Eq. (19),

$$
\begin{aligned}
& \left\langle\exp \left[-\Delta \int_{0}^{T} d u \eta(u)\right]\right\rangle=e^{f(T)}, \\
& f(T)=\Delta^{2} s^{2}[T / s-1+\exp (-T / s)] .
\end{aligned}
$$

Second, Eq. (21) contains the average

$$
\left\langle\eta\left(T_{1}\right) \exp \left[-\Delta \int_{0}^{T_{1}+T_{2}} d u \eta(u)\right]\right\rangle .
$$

By regarding $\Delta$ as a time-dependent quantity $\Delta(u)$, and using the functional derivative

$$
\frac{\delta}{\delta \Delta(v)} \Delta(u)=\delta(u-v)
$$

this can be rewritten as

$$
\begin{aligned}
\left\langle\eta\left(T_{1}\right) \exp \left[-\Delta \int_{0}^{T} d u \eta(u)\right]\right\rangle & =-\frac{\delta}{\delta \Delta\left(T_{1}\right)}\left\langle\exp \left[-\int_{0}^{T} d u \Delta(u) \eta(u)\right]\right\rangle \\
& =-\frac{\delta}{\delta \Delta\left(T_{1}\right)} \exp \left[\frac{1}{2} \int_{0}^{T} d u \int_{0}^{T} d v \Delta(u) \Delta(v)\langle\eta(u) \eta(v)\rangle\right] \\
& =-\Delta\left(T_{1}\right) \int_{0}^{T} d v e^{-\left|T_{1}-v\right| / s} \exp \left[\frac{1}{2} \int_{0}^{T} d u \int_{0}^{T} d v \Delta(u) \Delta(v) e^{|u-v| / s}\right],
\end{aligned}
$$


where $T=T_{1}+T_{2}$. By resetting $\Delta(t)=\Delta$, we can rewrite this as

$$
\begin{aligned}
& -\Delta \int_{0}^{T} d v e^{-\left|T_{1}-v\right| / s} \exp \left[\Delta^{2} \int_{0}^{T} d u(T-u) \exp (-u / s)\right] \\
& \quad=-\Delta s\left(2-\exp -T_{1} / s-\exp -T_{2} / s\right) e^{f(T)}
\end{aligned}
$$

In this way, Eq. (21) becomes

$$
\begin{aligned}
\overline{T_{i} T_{i+1}}= & \int_{0}^{\infty} d T_{1} \int_{0}^{\infty} d T_{2}\left[\lambda_{0}-\Delta^{2} s\left(2-e^{-T_{1} / s}-e^{-T_{2} / s}\right)\right] \\
& \times e^{-\lambda_{0} T+f(T)} .
\end{aligned}
$$

Finally, by transforming the variables from $\left(T_{1}, T_{2}\right)$ to $\left(T_{1}, T\right)$, the above integral is reduced to the single integral given in Eq. (22),

$$
\overline{T_{i} T_{i+1}}=\frac{1}{\lambda_{0}} \int_{0}^{\infty} d T\left[\lambda_{0} T-2 f(T)\right] e^{-\lambda_{0} T+f(T)} .
$$

[1] W.R. Softky and C. Koch, J. Neurosci. 13, 334 (1993).

[2] M.N. Shadlen and W.T. Newsome, Curr. Opin. Neurobiol. 4, 569 (1994).

[3] M.N. Shadlen and W.T. Newsome, J. Neurosci. 18, 3870 (1998).

[4] S. Shinomoto, Y. Sakai, and S. Funahashi, Neural Comput. 11, 935 (1999).

[5] Y. Sakai, S. Funahashi, and S. Shinomoto, Neural Networks 12, 1181 (1999).

[6] J.G. Nicholls, A.R. Martin, and B.G. Wallace, From Neuron to Brain, 3rd ed. (Sinauer, Sunderland, MA, 1992).

[7] D.R. Cox and P.A.W. Lewis, The Statistical Analysis of Series of Events (Methuen, London, 1966).

[8] Stochastic Point Processes: Statistical Analysis, Theory, and Applications, edited by P.A.W. Lewis (Wiley, New York, 1972).
[9] H.C. Tuckwell, Introduction to Theoretical Neurobiology (Cambridge University Press, Cambridge, England, 1988).

[10] T. Kaneko, Y. Kang, and N. Mizuno, J. Neurosci. 15, 8362 (1995).

[11] A.M. Thomson and J. Deuchars, Cereb. Cortex 7, 510 (1997).

[12] F. Gabbiani and C. Koch, Methods in Neuronal Modeling, 2nd ed. (The MIT Press, Cambridge, MA, 1999).

[13] T.M. Cover, and J.A. Thomas, Elements of Information Theory (Wiley, New York, 1991).

[14] C.M. Gray and D.A. McCormick, Science 274, 109 (1996).

[15] E.R. Kandel, J.H. Schwartz, and T.M. Jessell, Principles of Neural Science, 3rd ed. (Appleton \& Lange, London, 1991).

[16] P. Lansky, Phys. Rev. E 55, 2040 (1997).

[17] A.R. Bulsara, T.C. Elston, C.R. Doering, S.B. Lowen, and K. Lindenberg, Phys. Rev. E 53, 3958 (1996). 